\title{
FATORES PRECOCES ASSOCIADOS AOS COMPORTAMENTOS DE RISCO NA ADOLESCENCIA: UM ESTUDO DE COORTE POPULACIONAL
}

\section{Pôster}

Autores deste trabalho:

Ana Beatriz Bozzini: Departamento de Medicina Preventiva, Faculdade de Medicina USP, Universidade de São Paulo, Brasil anabeatrizbozzini@gmail.com

Rudi Rocha: Professor de Economia, Escola de Administração Pública - EAESP FGV, Fundação Getúlio Vargas, São Paulo, Brasil

Tiago N. Munhoz: Faculdade de Psicologia, Universidade Federal de Pelotas, Pelotas, Brasil

Iná S. Santos: Programa de Pós Graduação em Epidemiologia, Universidade Federal de Pelotas, Brasil

Aluísio JD Barros: Programa de Pós Graduação em Epidemiologia, Universidade Federal de Pelotas, Brasil

Alicia Matijasevich: Departamento de Medicina Preventiva, Faculdade de Medicina USP, Universidade de São Paulo, Brasil Programa de Pós Graduação em Epidemiologia, Universidade Federal de Pelotas, Brasil

Área do Trabalho: Pediatria

Data da submissão: $15 / 07 / 2018$ às $19 \mathrm{~h} 22 \mathrm{~min}$

\section{Justificativa}

Comportamentos de risco na adolescência afetam o desenvolvimento físico e emocional saudável do indivíduo. O conhecimento de seus determinantes precoces é de extrema importância para adotar medidas de prevenção.

\section{Objetivo(s)}

Este estudo avaliou em que medida o comportamento materno durante a gravidez e a saúde infantil perinatal e no primeiro ano de vida, associam-se a comportamentos de risco aos 11 anos (agressividade, comportamento depressivo, uso e abuso de álcool e tabaco)

\section{Método(s)}

Estudo longitudinal de coorte $(n=4231)$ em Pelotas, Brasil, com seguimento do nascimento até a adolescência inicial (11 anos). Realizou-se regressão logística através do programa Stata 13.0 para acessar associações estatisticamente significantes entre variáveis materno-infantis precoces e os comportamentos de risco na adolescência, ajustadas para variáveis demográficas.

\section{Resultado(s)}

Tabagismo materno durante a gravidez, pouco apoio do parceiro na gestação e história prévia do adolescente de hospitalização no primeiro ano de vida associaramse com envolvimento em brigas aos 11 anos (OR 1,73, IC95\%1,40-2,16; OR 1,44, IC95\%1,12-1,85 e OR 1,33, IC95\%1,04-1,70 respectivamente). Tabagismo materno 


\section{$4^{\circ}$ Congresso Internacional Sabaró \\ 13 a 15 de \\ 13 a 15 de
setembro de 2018}

Hotel Maksoud Plaza
Alameda Campinas, 150

durante a gravidez associou-se com envolvimento em brigas com armas aos 11 anos (OR 2,66, IC95\%1,31-5,39). Depressão materna durante a gestação e hospitalização do adolescente no primeiro ano de vida associaram-se com experimentação de cigarro aos 11 anos (OR 1,86, IC95\% 1,03-3,35 e OR 2,74, IC95\%1,52-4,94). Baixo peso ao nascer associou-se com uso recente de tabaco na adolescência (OR 16,23, IC95\%2,01-131,10). Baixo suporte emocional do parceiro na gestação, duração do aleitamento materno e hospitalização no primeiro ano de vida associaram-se com episódio depressivo aos 11 anos (OR 2,39, IC95\%1,00-5,70; OR 2,96, IC95\%1,28-6,86 e OR 2,46, IC95\%1,03- 5,87). Depressão materna na gestação associou-se com inatividade física do adolescente (OR 1,35, IC95\%1,04-1,75)

\section{Conclusão (ões)}

Os resultados demonstram que depressão e tabagismo maternos durante a gestação, baixo apoio emocional do parceiro na gravidez e hospitalização no primeiro ano de vida, foram os determinantes precoces mais associados a diversos comportamentos de risco no início da adolescência. Políticas públicas devem concentrar-se em reduzir depressão e tabagismo maternos, estimular o apoio dos parceiros durante a gestação, e prevenir as principais causas de hospitalização no primeiro ano de vida 